\title{
Inventories, Interest Rates, and Markups
}

\author{
David Glenn Bivin \\ Department of Economics, Indiana University Purdue University Indianapolis, Indianapolis, USA \\ E-mail:dbivin@iupui.edu \\ Received June 7, 2011; revised July 13, 2011; accepted July 25, 2011
}

\begin{abstract}
This note explains why inventories might rise with interest rates. Higher real interest rates not only increase the carrying cost of inventories they also reduce the present value of the markup on delayed sales. When the markup is large enough, it is profitable to increase stocks in order to avoid sales delays. Another possibility is that the firm has an incentive to smooth its total stocks so that an increase in the real interest rate causes finished goods to fall but the reduction is partially offset by an increase in raw materials.
\end{abstract}

Keywords: Inventories, Interest Rates

\section{Introduction}

An enduring puzzle in the inventory literature is that inventories do not appear to decline when the real interest rate rises. Inventories provide a bridge between the time that stocks are delivered, the time they are processed, and the time they are sold. During this time span, the purchase and/or processing cost must be financed. When the real interest rate rises, the firm has an apparent incentive to speed up the process in order to receive the revenue to pay off these financing costs more rapidly. The idea is intuitive. Indeed, Blinder and Maccini [1] capture the view of the profession when they state that "the idea is so simple that it is hard to imagine how it can be wrong" (p. 82). ${ }^{1}$

The empirical evidence provides only sparse support for the hypothesis. ${ }^{2}$ Recently, Maccini, Moore, and Schaller [2] developed an innovative test for a long-run inverse relationship between the interest rate and finished goods inventories in the context of the linear quadratic model. Their estimates suggest a significant inverse relationship in a number of non-durables finished goods for the time period examined. Bivin [4] extends the model to account for durables producers and work-in-process and raw ma-

\footnotetext{
${ }^{1}$ In fairness, they also point that interest rates influence inventories through a number of channels including the discounting of revenue. That is the crux of the models developed in sections 2 and 3.

${ }^{2}$ Maccini, Moore, and Schaller [2] provide an excellent review of the literature and point out that interest in the topic seems to have waned. Ramey and West [3] survey some of the more prominent work in the field. Their results suggest that significant inventory responses typically occur in less than $25 \%$ of the models that allow for an interest rate effect (Table 11, p. 907).
}

terials inventories, as well as the more recently available NAICS data. Among this larger set of cases, the results are far less robust.

This paper adopts a different strategy from that of the empirical literature. The goal here is to explain why inventories might actually rise in response to an increase in the real interest rate. The key ingredient is backorders that provide the firm with a second opportunity to make a sale when the first opportunity is lost due to inadequate stocks. The reward to filling a backorder is smaller than the reward for filling an order when it first arrives due to discounting. While a higher real interest rates raises inventtory-carrying cost, it also raises the penalty for failing to fill a new order.

The model is similar in spirit to that of Kahn [5]. Kahn's model features explicit stock outs of finished goods and random demand. One of the versions of his model allows backorders and in that model finished goods are independent of the real interest rate. It is shown here that adding a carrying cost of finished goods to the model causes the firm's safety stock to rise as the real interest rate rises. A model that is similar in spirit is then applied to the case of a firm that holds only raw materials.

A third model demonstrates why raw materials might rise in the context of a linear-quadratic model with inventtories of both finished goods and raw materials. In this case, the increase is due to an effort to smooth total stocks.

\section{Backorders, Finished Goods and Carrying Cost}

Output available for sale is equal to finished goods on 
hand at the beginning of the period plus output produced during the period. Sales are the minimum of demand and output available for sale:

$$
S=\min \left(Q, X+F_{-1}\right)
$$

where $S$ is sales, $Q$ is demand, $X$ is output, and $F$ is the end-of-period stock of finished goods. $F_{-1}$ is finished good inventory inherited from the prior period. Unfilled orders are backlogged and added to total demand in the following period. Total demand is

$$
Q=\bar{Q}+\left(Q_{-1}-S_{-1}\right)+\sigma \varepsilon
$$

where $\bar{Q}$ and $\sigma$ are positive constants and $\varepsilon$ is a standard normal variate. The demand shock is observed after the output decision has been made. Backorders are added to new orders to yield total demand. Finished goods evolve as

$$
F=F_{-1}+X-S
$$

The cost function is linear. The decision variable is output and the solution satisfies the Bellman equation:

$$
\max _{\mathrm{X}} V\left(F_{-1}\right)=E\left[p S-c X-b_{F} F+\lambda V\left(F_{-1}+X-S\right)\right]
$$

where $p, c$, and $b_{F}$ are the sales price, per unit production cost, and the per-unit carrying cost for finished goods, respectively. The parameter $\lambda$ is the single-period discount factor defined as $\lambda=1 /(1+r)$ where $r$ is the real interest rate.

Let $\pi$ denote the probability that demand is satisfied:

$$
\begin{aligned}
\pi & =\operatorname{Pr}\left[F_{-1}+X \geq \bar{Q}+\left(Q_{-1}-S_{-1}\right)+\sigma \varepsilon\right] \\
& =\Phi\left[\frac{\left(F_{-1}+X\right)-\bar{Q}-\left(Q_{-1}-S_{-1}\right)}{\sigma}\right]
\end{aligned}
$$

where $\Phi(\bullet)$ is the cumulative standard normal density. Taking the first derivative of (4) with respect to $X$ yields

$$
E\left(V_{X}\right)=(1-\pi)(1-\lambda) p-c-\pi b_{F}+\lambda E\left(V_{F}\right)=0
$$

Since output is immediately available for sale, finished goods on hand and current output are perfect substitutes and the shadow value of finished goods is simply $E\left(V_{F}\right)$ $=c$. Substituting this result into (6) and solving for $\pi$ yields.

$$
\pi=\frac{(1-\lambda)(p-c)}{(1-\lambda)\left(p+b_{F}\right)}=\frac{(p-c)}{p+\frac{1}{(1-\lambda)} b_{F}}=\frac{(p-c)}{p+\frac{1+r}{r} b_{F}}
$$

If $b_{F}=0$ this expression is identical to that in Kahn [5]. As $b_{F}$ rises, $\pi$ declines implying a greater willingness to delay a sale. The derivative of (7) with respect to $r$ is

$$
\frac{\partial \pi}{\partial r}=\frac{b_{F}(p-c)}{\left[r p+(1+r) b_{F}\right]^{2}}
$$

This expression is clearly positive as long as $b_{F}>0$ and it increases as $p-c$ rises. This supports the contention that the counterintuitive behavior is driven by the markup. Solving for $X+F_{-1}$ from (5) yields

$$
X+F_{-1}=E(Q)+\sigma \Phi^{-1}(\pi)=E(Q)+\sigma k_{X}
$$

where $k_{X}=\Phi^{-1}(\pi)$. In the steady-state, $X=E(Q)$ and $\sigma k_{X}$ is target finished goods (when $k_{X}>0$ ) or backorders (when $k_{X}<0$ ). Since $k_{X}$ is an increasing function of $\pi$, it follows that target finished goods are an increasing function of $r$. The reason is that the implicit cost of backorders rises when the real interest rate rises because the markup is discounted more heavily. Thus the firm has an incentive to raise its safety stock as a means of economizing on this penalty.

\section{Production to Order ${ }^{3}$}

Under production to order, goods are customized in some sense and production cannot commence until the order arrives. Thus the current demand shock is known prior to the production decision. Here, the firm accumulates homogeneous raw materials in anticipation of orders. It has an incentive to do so because of a delivery lag that prevents newly ordered materials from arriving early enough in the period to be processed and sold by the end of the period. As a result of the delivery lag, the firm targets it deliveries on fulfilling unmet demand in the current period along with new orders that are expected to arrive in the following period. The buffer against demand shocks is now fulfilled by raw materials rather than finished goods. When the buffer is exhausted, output is starved and the firm accumulates backorders until the following period. Since the firm observes the demand shock prior to placing its materials orders it knows at that time the level of backorders it will have on hand at the end of the period.

Inputs are defined such that one unit of raw material is required for each unit of output. The firm has an incentive to fill its orders as soon as possible and so output is defined as ${ }^{4}$

$$
X=\min \left(Q, M_{-1}\right)
$$

where $M_{-1}$ is raw materials on hand at the end of the prior period. Demand in the following period is now defined as

$$
Q_{1}=\bar{Q}+\max \left(Q-M_{-1}, 0\right)+\sigma \varepsilon_{1}
$$

\footnotetext{
${ }^{3}$ The model in this section is similar to that of Kahn [6] and Bivin [7]. Both assume the presence of unfilled orders, raw materials, and binding stock out constraints.

${ }^{4}$ It will not be profitable to produce more than total demand when the cost of finished goods is sufficiently high and/or the final products are so individualized that the demand for the specific product produced is unlikely to eventually appear. For a model of the production-to- stock/ production-to-order decision see Krane [8].
} 
Raw materials evolve as

$$
M=M_{-1}+D-X
$$

where $D$ is the delivery of raw materials in the current period.

Based on results from previous models of this type, we posit the following solution:

$$
M^{*}=E_{0}\left(Q_{1}\right)+\sigma k_{D}=\bar{Q}+\max \left(Q-M_{-1}, 0\right)+\sigma k_{D}
$$

where the inventory buffer is now captured by $\sigma k_{D}$. It then follows that $\operatorname{Pr}\left(M^{*} \geq Q_{1}\right)=\operatorname{Pr}\left(k_{D} \geq \varepsilon\right)$. As before, this probability will be denoted as $\pi$. The inventory rule implies a constant probability of unmet orders. This is consistent with the linear cost function described below.

From (13) it follows that

$$
\begin{aligned}
D^{*} & =M^{*}+X-M_{-1} \\
& =\bar{Q}+\max \left(Q-M_{-1}, 0\right)+\sigma k_{D}+\min \left(Q, M_{-1}\right)-M_{-1} \\
& =\bar{Q}+Q-M_{-1}+\sigma k_{D}
\end{aligned}
$$

Note that a one unit increase in $M_{-1}$ yields a one unit decrease in $D^{*}$ regardless of whether the extra unit of materials is used in current production. Intuitively, $D^{*}+$ $M_{-1}$ are total materials available for production dur- ing the current and following periods. These stocks are targeted on current actual demand plus next period's expected demand and a safety margin. Demand not met in the current period is added to next period's demand so that total demand over the next two periods is independent of $M_{-1}$.

Output in the following period may now be defined as

$$
\begin{aligned}
X_{1} & =\min \left(Q_{1}, M^{*}\right) \\
& =\bar{Q}+\max \left(Q-M_{-1}, 0\right)+\sigma \min \left(k_{D}, \varepsilon_{1}\right)
\end{aligned}
$$

The significance of (15) is that next period's output depends upon last period's inventories.

Costs are still linear and the objective function is

$$
\max _{D} V\left(M_{-1}\right)=(p-c) X-v D-b_{M} M+\lambda E\left[V\left(M_{-1}+D-X\right)\right]
$$

where $v$ is the purchase price for one unit of raw materials and $b_{M}$ is the per-unit carrying cost of raw materials. The first-order condition requires

$$
E\left(\frac{\partial V}{\partial D}\right)=-\left(v+b_{M}\right)+\lambda E\left(V_{M}\right)
$$

The marginal shadow value of raw materials is that it enables the firm to sell its output sooner. By the envelope theorem:

$$
\begin{aligned}
E\left(\frac{\partial V\left(M_{-1}\right)}{\partial M_{-1}}\right) & =(p-c) E\left(\frac{\partial X}{\partial M_{-1}}\right)-v E\left(\frac{\partial D}{\partial M_{-1}}\right)-b_{M} E\left(\frac{\partial M}{\partial M_{-1}}\right) \\
& +\lambda(p-c) E\left(\frac{\partial X_{1}}{\partial M_{-1}}\right)-\lambda v E\left(\frac{\partial D_{1}}{\partial M_{-1}}\right) \\
& -\lambda\left[b_{M}-E\left(\frac{\partial V\left(M_{1}\right)}{\partial M_{-1}}\right)\right] E\left(\frac{\partial M_{1}}{\partial M_{-1}}\right)
\end{aligned}
$$

According to the model, the firm fulfills all of its orders in the period in which they arrive with probability $\pi$. Those orders not filled in the period in which they arrive are fulfilled in the following period. Thereafter, $M_{-1}$ is irrelevant for the firm's performance. The decision for $D_{l}$ is independent of $M_{-1}$ and so is $M_{1}$. Therefore, based on (13) through (15), it follows that

$$
\begin{aligned}
& E\left(\frac{\partial X}{\partial M_{-1}}\right)=E\left(\frac{\partial X_{1}}{\partial M_{-1}}\right)=1-\pi, E\left(\frac{\partial D}{\partial M-1}\right)=-1, \\
& E\left(\frac{\partial M}{\partial M_{-1}}\right)=-\pi, E\left(\frac{\partial D_{1}}{\partial M_{-1}}\right)=E\left(\frac{\partial M_{1}}{\partial M_{-1}}\right)=0
\end{aligned}
$$

and it follows that (18) may be rewritten as

$$
E\left(V_{M}\right)=\left[(p-c)(1-\lambda)+b_{M}\right](1-\pi)+v
$$

Substituting (19) into (17) and rearranging yields

$$
\frac{v+b_{M}}{\lambda}=\left[(p-c)(1-\lambda)+b_{M}\right](1-\pi)+v
$$

Solving for $\pi$.

$$
\pi=\frac{p-c-\lambda^{-1}\left(v+b_{M}\right)}{(p-c)+b_{M}(1-\lambda)^{-1}}
$$

The expression is a legitimate probability as long as $p-c-\lambda^{-1}\left(v+b_{M}\right)>0$. This expression is the per-unit markup when materials are purchased this period and processed and sold in the following period. Therefore it is non-negative. The influence of a change in the real interest rate on $\pi$ is not apparent from the expression but it can be shown that $\partial \pi \partial r>0$ if

$$
p-c-v-b_{M}>\frac{(1-\lambda)^{2}}{\lambda^{2}} \frac{\left(v+b_{M}\right)}{b_{M}}\left(p-c+b_{M}\right)
$$

The expression on the right is a "quasi-markup" with raw materials used up in production valued at their replacement cost. It is larger than the markup defined earlier and thus must be positive. As long as this markup is sufficiently high, $k_{D}$ increases when the real interest rate rises. For $\lambda$ near one, the expression on the right-hand side of (22) is likely to be small. It is worth noting that this result holds even when $b_{M}=0$.

Since $k_{D}=\Phi^{-1}(\pi)$, it follows from (13) that $M^{*}$ is an increasing function of $r$ when $\pi$ is an increasing function of $r$.

\section{Multi-Stage Stocks in the Linear- Quadratic Model ${ }^{5}$}

Finally, it is straightforward to show that steady-state raw materials may rise in the linear quadratic model in which increasing costs are assigned to finished goods,

\footnotetext{
${ }^{5}$ The model in this section is based upon Humphreys, et al. [9] and Bivin [4].
} 
raw materials, and total stocks. The last cost, which to my knowledge has not been introduced before, carries some intuition. It suggests that costs are lower for a firm with an excess unit of finished goods and a one-unit shortage of raw materials than for a firm with an excess unit of both finished goods and raw materials. This could be the case if finished goods and raw materials share the same warehouse space. Also, in a more general model than that presented here, the price response to inventory disequilibria may be smaller the closer that total stocks are to their equilibrium.

Consider a production-to-stock firm in which sales are equal to news orders. Sales are typically random and exogenous but here they are treated as a known constant along with the remaining exogenous variables. ${ }^{6}$ The objective function is

$$
\begin{aligned}
\text { Cost }= & \sum_{t=0}^{\infty} \lambda^{k}\left\{c X_{t}^{2}+w X_{t}+v D_{t}\right. \\
& +b_{F}\left(F_{t-1}-\delta_{F} S\right)^{2}+b_{M}\left(M_{t-1}-\delta_{M} S\right)^{2} \\
& \left.+b_{I}\left[\left(M_{t-1}+F_{t-1}\right)-\delta_{I} S\right]^{2}\right\}
\end{aligned}
$$

where $c, b_{F}, b_{M}, b_{I}, w>0$ and $\delta_{F}, \delta_{M}, \delta_{I} \geq 0$, and $w$ is unit labor cost. As before it is assumed that each unit of out-put requires one unit of raw materials. The firm minimizes (23) with respect to $X_{t}$ and $D_{t}$ subject to the inventory investment identities:

$$
\begin{gathered}
F_{t}-F_{t-1}=X_{t}-S_{t} \\
M_{t}-M_{t-1}=D_{t}-X_{t}
\end{gathered}
$$

The Lagrangian form of the cost minimization problem is

$$
\begin{aligned}
\operatorname{Cos} t=\sum_{t=0}^{\infty} \lambda^{k}\left\{c X_{t}^{2}\right. & +w X_{t}+v D_{t}+b_{F}\left(F_{t-1}-\delta_{F} S\right)^{2} \\
& +b_{1}\left[\left(M_{t-1}+F_{t-1}\right)-\delta_{1} S\right]^{2} \\
& +\phi_{F, t}\left(F_{\mathrm{t}}-F_{t-1}-X_{t}+S\right) \\
& \left.+\phi_{M, t}\left(M_{t}-M_{t-1}-D_{t}+X_{t}\right)\right\}
\end{aligned}
$$

where $\phi_{F, t}$ and $\phi_{M, t}$ are Lagrangian multipliers. In addition to the inventory investment identities, the first-order condition requires

$$
\begin{aligned}
& \frac{\partial \operatorname{Cos} t}{\partial X_{t}}=0.5 c X_{t}+w-\phi_{F, t}+\phi_{M, t}=0 \\
& \frac{\partial \operatorname{Cos} t}{\partial D_{t}}=v-\phi_{M, t}=0
\end{aligned}
$$

\footnotetext{
${ }^{6}$ This is merely for convenience. Even if sales are random, certainty equivalence applies and the optimal solution can be found by replacing the random variables with their expected values. The influence of uncertainty on inventories is built into the model through the specification of the inventory target functions. The conclusions do not depend upon this specification of the targets.
}

$$
\begin{aligned}
\frac{\partial \text { Cost }}{\partial F_{t}}= & 0.5 b_{F} \lambda\left(F_{t}-\delta_{F} S\right) \\
& +0.5 b_{I} \lambda\left(F_{t}+M_{t}-\delta_{I} S\right)+\phi_{F, t}-\lambda \phi_{F, t+1} \\
= & 0 \\
\frac{\partial \operatorname{Cost} t}{\partial M_{t}}= & 0.5 b_{M} \lambda\left(M_{t}-\delta_{M} S\right) \\
+ & 0.5 b_{I} \lambda\left(F_{t}+M_{t}-\delta_{I} S\right)+\phi_{M, t}-\lambda \phi_{M, t+1}=0
\end{aligned}
$$

Our interest is in the steady-state in which $D^{*}=X^{*}=S$, $\phi_{F, t}=\phi_{F}$, and $\phi_{M, t}=\phi_{M}$. From (27b) it is apparent that $\phi_{M}$ $=v$ and it follows from (27a) that

$$
\phi_{F}=0.5 c S+v+w
$$

This is the marginal replacement cost of finished goods in the steady state.

Note that $(1-\lambda) \phi_{F} / \lambda=r \phi_{F}$ and $(1-\lambda) \quad \phi_{M} / \lambda=r \phi_{M}$. Equations $(27 \mathrm{c})$ and $(27 \mathrm{~d})$ may be written as

$$
\begin{aligned}
& 0.5\left(b_{F}+b_{I}\right) F^{*}+0.5 b_{I} M^{*} \\
= & 0.5\left[b_{F} \delta_{F}+b_{I} \delta_{I}-0.5 c r\right] S-r(v+w) \\
& 0.5\left(b_{M}+b_{I}\right) M^{*}+0.5 b_{I} F^{*} \\
= & 0.5\left[b_{M} \delta_{M}+b_{I} \delta_{I}\right] S-r v
\end{aligned}
$$

Solving:

$$
\begin{aligned}
& F^{*}=\theta_{F} S-r \frac{b_{M}(0.5 c S+w+v)+b_{I}(0.5 c S+w)}{0.5 T} \\
& M^{*}=\theta_{M} S-r \frac{b_{F} v-b_{I}(0.5 c S+w)}{0.5 T}
\end{aligned}
$$

where $T=\left(b_{M}+b_{I}\right)\left(b_{F}+b_{I}\right)-b_{I}^{2}=b_{M} b_{F}+b_{I}\left(b_{M}+b_{F}\right)>$ 0 . Neither $\theta_{F}$ nor $\theta_{M}$ depend upon the real interest rate. When $b_{I}=0$ both stocks fall when the real interest rate arise and, from (30), finished goods fall regardless of the value of $b_{I}$. Moreover, adding (30) and (31) together:

$$
\begin{aligned}
F^{*}+M^{*} & =\left(\theta_{F}+\theta_{M}\right) S \\
& -r \frac{b_{M}[0.5 c S+w+v]+b_{F} v}{0.5 T}
\end{aligned}
$$

and it is clear that total stocks decline when the real interest rate rises.

However, according to $(31), M^{*}$ rises if $b_{I}$ is sufficiently large relatively to $b_{F}$. Specifically, an increase in $r$ causes $M^{*}$ to rise if

$$
\frac{b_{I}}{b_{F}}>\frac{v}{0.5 c S+w}
$$

The numerator of the expression on the right is the marginal cost of a delivery in the steady state and the denominator is the marginal cost of a unit of output in the steady state. If the latter is sufficiently large relative to the former, the firm will respond to an increase in the real interest rate by reducing its finished goods and raising its raw materials to maintain overall stock equilibrium. 
Given the choice, the firm disinvests in finished goods rather than raw materials because finished goods incorporate processing costs (which must be financed) while raw materials do not. Thus, an increase in the real interest rate effectively delays production leading the firm to accumulate pre-production stocks at the expense of post-production stocks.

\section{Conclusions}

This paper presents three scenarios in which finish goods or raw materials may rises in response to an increase in the real interest rate. In the first two, production lags, backorders, and stockout constraints are responsible for the counter-intuitive result: the firm accumulates additional stocks despite the increased carrying cost in order to avoid delaying its revenue which, of course, is also discounted. In the third scenario, the firm is subject to a cost assigned to its total stocks. When the real interest rate rise, the firm reduces finished goods but, under certain combinations of costs, raises raw materials as a means of smoothing total stocks. The interesting feature of these conclusions is that they are generated within the context of standard models and require only straight forward assumptions.

\section{References}

[1] A. S. Blinder and L. J. Maccini, "Taking Stock: A Critical Assessment of Recent Research on Inventories," Journal of Economic Perspectives, Vol. 5, No. 1, 1991, pp. 73-96.

[2] L. J. Maccini, B. J. Moore and H. Schaller, "The Interest Rate, Learning, and Inventory Investment," American Economic Review, Vol. 94, No. 5, 2004, pp. 1303-1327. doi:10.1257/0002828043052295

[3] V. A. Ramey and K. D. West, "Inventories," In: J. B. Taylor and M. Woodford, Handbook of Macroeconomics, Vol. 1B, Elsevier, New York City, 1999, pp. 863-923.

[4] D. G. Bivin, "Inventories and Interest Rates: A Stage of Fabrication Approach," The Berkeley Electronic Journal of Macroeconomics, Vol. 10, No. 1, 2010.

[5] J. A. Kahn, "Inventories and the Volatility of Production," American Economic Review, Vol. 77, No. 4, 1987, pp. 667-679.

[6] J. A. Kahn, "Durable Goods Inventories and the Great Moderation," Federal Reserve Bank of New York Staff Report, New York, No. 325, May 2008.

[7] D. G. Bivin, "Production Chains and Output Volatility," Unpublished, Department of Economics, Indiana University Purdue University Indianapolis.

[8] S. D. Krane, "The Distinction Between Inventory Holding and Stockout Costs: Implications for Target Inventories, Asymmetric Adjustment, and the Effects of Aggregation of Production Smoothing," International Economic Review, Vol. 35, No. 1, 1994, pp. 117-136. doi: $10.2307 / 2527093$

[9] B. R. Humphreys, L. J. Maccini and S. Schuh, "Input and Output Inventories," Journal of Monetary Economics, Vol. 47, No. 2, 2001, 347-375. doi:10.1016/S0304-3932(01)00046-0 